\title{
Adjoint Monte Carlo Simulation of Fusion Product Activation Probe Experiment in ASDEX Upgrade tokamak
}

\author{
Simppa Äkäslompolo* \\ Aalto University, Finland \\ E-mail: simppa.akaslompolo@alumni.aalto.fi
}

\section{Georges Bonheure}

ERM-KMS, Brussels, Belgium ${ }^{\dagger}$

\section{Giovanni Tardini}

Max-Planck-Institut für Plasmaphysik, Garching, Germany

\section{Taina Kurki-Suonio}

Aalto University, Finland

\section{The ASDEX Upgrade team}

\begin{abstract}
The activation probe is a robust tool to measure flux of fusion products from a magnetically confined plasma. A carefully chosen solid sample is exposed to the flux, and the impinging ions transmute the material makig it radioactive. Ultra-low level gamma-ray spectroscopy is used post mortem to measure the activity and, thus, the number of fusion products.

This contribution presents the numerical analysis of the first measurement in the ASDEX Upgrade tokamak, which was also the first experiment to measure a single discharge. The ASCOT suite of codes was used to perform adjoint/reverse Monte-Carlo calculations of the fusion products. The analysis facilitated, for the first time, a comparison of numerical and experimental values for absolutely calibrated flux. The results agree to within a factor of two, which can be considered a quite good result considering the fact that all features of the plasma cannot be accounted in the simulations.
\end{abstract}

Also an alternative probe orientation was studied. The results suggest that a better optimized orientation could measure the flux from a significantly larger part of the plasma.

First EPS Conference on Plasma Diagnostics - 1st ECPD,

14-17 April 2015

Villa Mondragone, Frascati (Rome) Italy

\footnotetext{
${ }^{*}$ Speaker.

${ }^{\dagger}$ Present address: European Commision, Research Directorate General
} 


\section{Introduction}

In quest of an escaping fast ion measurement diagnostics applicable for ITER, the fusion product flux at the midplane manipulator in ASDEX Upgrade (AUG) was measured for the first time [1] using an activation probe [2]. This contribution presents numerical analysis of these experiments using the ASCOT code [3]. The fusion reactivity is calculated using ASCOT and its AFSI Fusion Source Integrator component, and for verification purposes, also with TRANSP [4]. The detailed probe geometry was included in the AUG 3D first wall model. We performed an adjoint Monte Carlo simulation, that was equivalent to following the collisionless orbits of the fusion products (mainly protons but also tritons and helium-3) from their birth location in the plasma to the probe. In the adjoint method, markers are launched backward in time from the probe towards the plasma. The main result of this contribution is the comparison of measured and simulated fusion product flux into the probe. The numerical analysis provides, for the first time, absolutely calibrated flux of fusion products to the probe. Previous analysis [2], performed on JET measurements, has only provided profiles of the flux as a function of major radius, which has needed external calibration. The measurements and current analysis are in reasonable quantitative agreement. The analysis was performed also for an alternative probe geometry, which showed higher fluxes in a previous numerical study [5].

\section{Materials and Methods}

An activation probe exposes carefully chosen material samples to the escaping fusion product flux from the plasma. The high-energy products then transmute part of the target material making it radioactive. The resulting activity can be measured post-mortem with ultralow level gamma-ray spectroscopy.

The probe was connected to the midplane manipulator near the outer midplane (figure 1) of the ASDEX Upgrade tokamak. The probe held six $\sim 1 \mathrm{~cm}^{2}$ sized samples as illustrated in figure 2(a). The two $\mathrm{B}_{4} \mathrm{C}$ samples (second and fourth, when counting from the tip of the probe), are of interest for this study. The flux of protons to those samples from the $\mathrm{D}(\mathrm{D}, \mathrm{T}) \mathrm{p}$ reaction was successfully measured, and is the subject of comparisons between measurement and simulation. The sample holder was inside a protective graphite cap, which has a narrow slit allowing fusion products to hit the samples (figure 2(b)). The slit was situated at the top of the graphite cap.

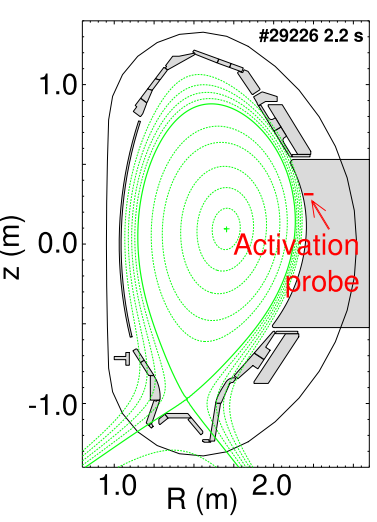

Figure 1: Plasma crosssection with the probe location indicated.

In the numerical analysis, the model of the probe was included in the detailed 3D wall (figure 2(c)) of the tokamak. The general shape and dimensions of the graphite cap and sample holder with samples are included in the model. The overall shape and cross-sections of the model are shown in figure $2(\mathrm{~d}-\mathrm{e})$.

In the experiment, the probe was exposed to the dedicated ASDEX Upgrade discharge \#29226. It was a deuterium plasma with $7.25 \mathrm{MW}$ of injected deuterium neutral beam power. The plasma profiles were acquired using integrated data analysis [6]. The core temperature was approximately 
(a)

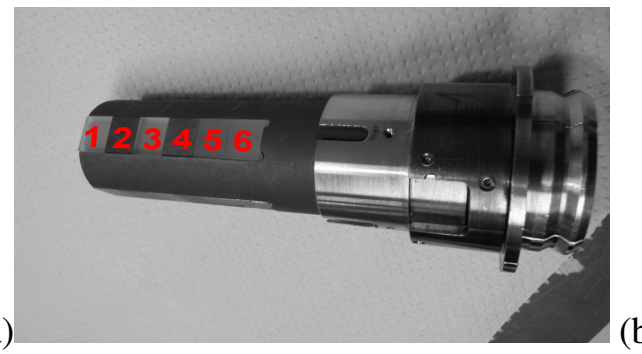

(b)

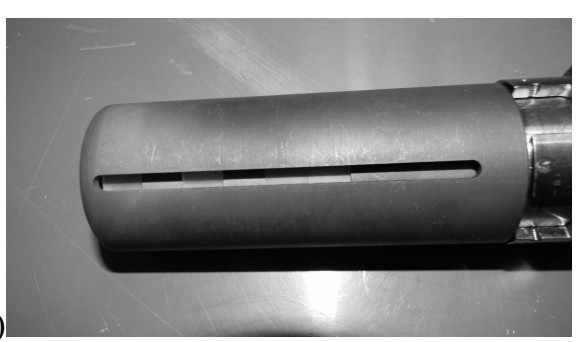

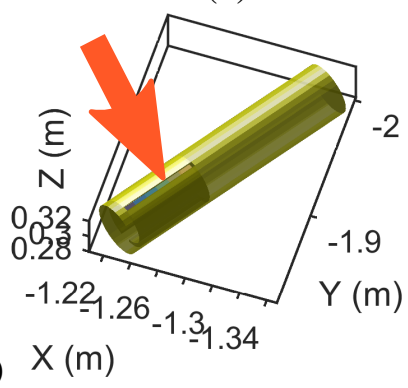

(d)

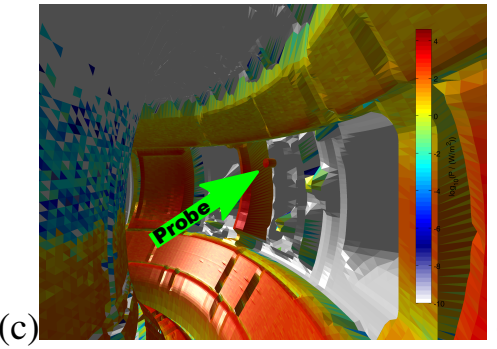

Figure 2: Illustration of the probe. (a) The sample holder with samples numbered. (b) The graphite cap covering the sample holder. (c) The 3D-wall model, including a model for the probe. The coloring indicates fusion product flux distribution onto the first wall as calculated by forward Monte-Carlo [5]. (d) Close up of the probe geometry used in this study. The "passive" cover is shown in semi-transparent yellow. The samples are partly visible through the slit (indicated) and each has unique color. (e) Cross-sectional view of the probe. The green mesh is used at the ends of the probe and the blue mesh is used at the ends of the cavity between the sample holder and the graphite cap. The samples and the opening in the cover are at the far right side.

$3 \mathrm{keV}$ and density approximately $7 \cdot 10^{19} \mathrm{~m}^{-3}$. In the modelling, nitrogen was used as the effective impurity, to match the measured effective charge number. The plasma shape and the probe location is shown in figure 1 . The plasma flat top was approximately $5 \mathrm{~s}$ in length.

\subsection{Adjoint integration scheme}

The roles of the relatively large source, the plasma, and the tiny target, i.e the samples within the probe, are exchanged in the adjoint Monte-Carlo integration. Since the target is small (and only visible through the narrow slit in the graphite shell) and the source is large, only very few markers launched from the plasma will find their way to the target. Therefore, most of the markers do not contribute to the integral. In the adjoint method, markers start backward in time from the target and are much more likely to pass through the plasma.

In practise, the calculation is done in two phases. First the fusion product source rate is calculated and then markers are followed backward in time from the samples to the plasma. Both calculations are done assuming steady state.

The calculation of fusion reactivity started with the calculation of the NBI ionisation with the BBNBI code [7]. Then, the slowing down distribution was calculated with ASCOT [3], taking into account collisions between the background plasma and the slowing down guiding centres of beam deuterons. The fusion reactivity was finally calculated using the plasma profiles and slowing down distributions with the AFSI Fusion Source Integrator, a part of the ASCOT suite of codes. The reactivity was stored in a $(R, z)$ (major radius, elevation) grid. A match within a few percents was 
found when comparing fusion reactivities calculated by AFSI and TRANSP [4] when using the identical plasma profiles and slowing down distributions.

In the second phase of the calculation, the markers were followed backwards in time. The initial marker distribution was uniform on the targets and the marker velocity distribution was assumed isotropic. The full gyro motion of the particle orbits was followed backwards in time until they hit the probe structures or an other part of the tokamak wall. The adjoint density of the markers was calculated in a similar grid as the reactivity. The flux of fusion products arriving at the target was then optained by multiplying the two grids element-wise and summing up. For full details, please see [8].

\section{Results}

The calculation of flux was done in three phases, calculation of the fusion reactivity, calculation of the adjoint density and multiplication of these two together. The fusion reactivity was calculated for thermonuclear, beam-target and beam-beam fusions. The sum of these three reactions is shown in figure $3(a)$ for the reaction $\mathrm{D}(\mathrm{D}, \mathrm{p}) \mathrm{T}$ (The other reactivity, $\mathrm{D}\left(\mathrm{D},{ }^{3} \mathrm{He}\right) \mathrm{n}$ is nearly identical.)

The adjoint calculation was performed by launching three million markers from the samples. Approximately $15 \%$ of the markers exited the the graphite cap through the slit. Most markers hit the wall in less than a microsecond, and even the last one within 100 microseconds. During their short flights, they produced the density shown in 3(b). The few long-lived markers produce the low density area reaching to the centre of the plasma. The adjoint density is closely related to the "instrument function" of the probe: It directly indicates which parts of the plasma the probe measures and with what kind of relative sensitivity.

The total flux to targets was obtained by multiplying the fusion reactivity and adjoint density $(R, z)$ grids point-wise together (figure 3(c)), (and scaling with the relevant factors). The flux was the sum over the grid. The resulting flux is presented in figure 4(a). The experimentally measured total number of particles with measurement uncertainty was divided by the flat top length of $5.0 \mathrm{~s}$ to calculate the number of arriving particles per second. A TRANSP simulation of the discharge was performed to verify the forward part of the calculation and to asses of the simulation errors. The calculation was repeated for three time steps from the TRANSP simulation. The four calculations are within a factor of two of the experimental measurement (figure 4).

Previous, regular Monte Carlo study [5] suggested that by rotating the probe by $90^{\circ}$, the fusion product flux would have been much higher, because many more products hit the sides of the probe than the top (figure 5(a)). This was studied with the adjoint method, and indeed, rotating the probe by $90^{\circ}$ would have strongly changed the instrument function (figure 3(d)). Fusion reactivity at the core would have had a larger contribution (figure 3(e)). However, the signal would have increased only in certain samples (figure 5(b)).

\section{Summary and Discussion}

We have shown that our computational tools can produce quantitatively good results when modelling the activation probe in ASDEX Upgrade. The modelling could be improved, by includ- 


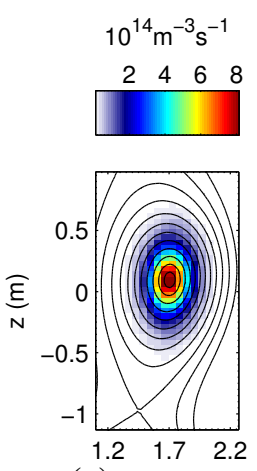

(a) $\mathrm{R}(\mathrm{m})$

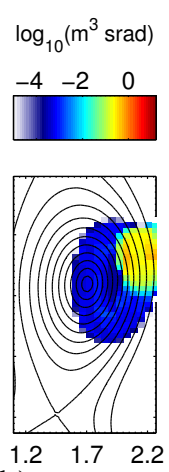

(b) $\mathrm{R}(\mathrm{m})$

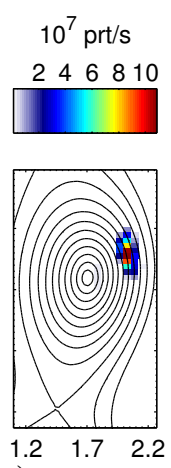

(c) $R(m)$

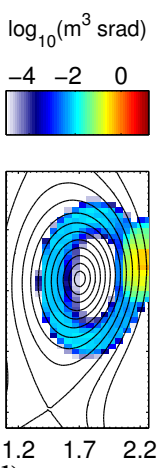

(d) $\mathrm{R}(\mathrm{m})$
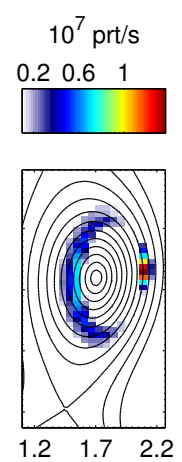

(e) $\mathrm{R}(\mathrm{m})$

Figure 3: (a) The fusion reactivity in $(R, z)$ grid. (b) The adjoint marker density of protons, summed up for all samples. (c) The origin of the contributing particles. (d) The adjoint marker density of protons in case of $90^{\circ}$ rotation of the probe. (e) The origin of the contributing particles. $\left(90^{\circ}\right.$ rot.)

(a)

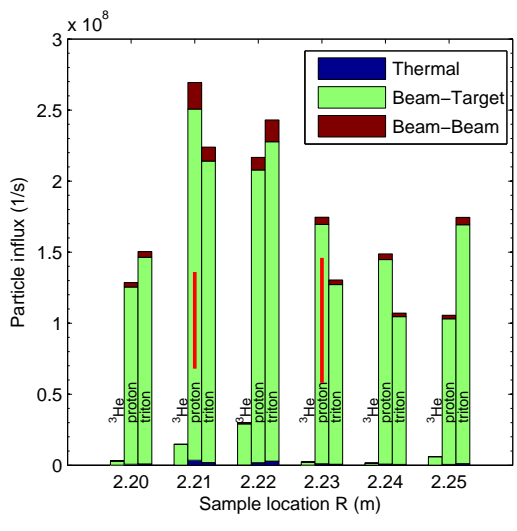

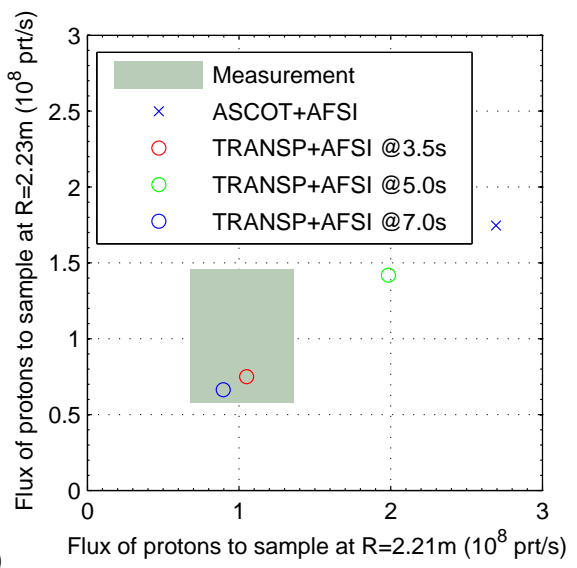

(b)

Figure 4: (a) Simulated and measured flux to the samples. In each group of bars, the left one is ${ }^{3} \mathrm{He}$, the middle one protons and the right one tritons. The red bar is the measured proton flux with uncertainty. (b) Measurements and adjoint simulation results from various fast ion densities and profiles presented in a 2D plot.

ing e.g. some of the following features neglected here: the simulations were performed for single time-slice while the plasma evolves in time. The fusion products were assumed to be collisionless. Their birth distribution was assumed to be isotropic and monoenergetic, which is certainly not true for beam-target fusion products. Regardless of these simplifications, the calculated fusion product flux to the probe was within $40 \%$ of the experimentally measured uncertainty.

The analysis of rotated probe head suggests that there is large room for optimising the measurement geometry and this contribution describes the tool for the studies. One could conceivably design a probe that is sensitive only to a specific part of plasma. It would be interesting to study the effect of pushing the probe nearer the plasma, rotating the probe, changing the slit shape etc. These studies could also be done for ITER, for which the robustness of the activation probe offers unique possibilities. 

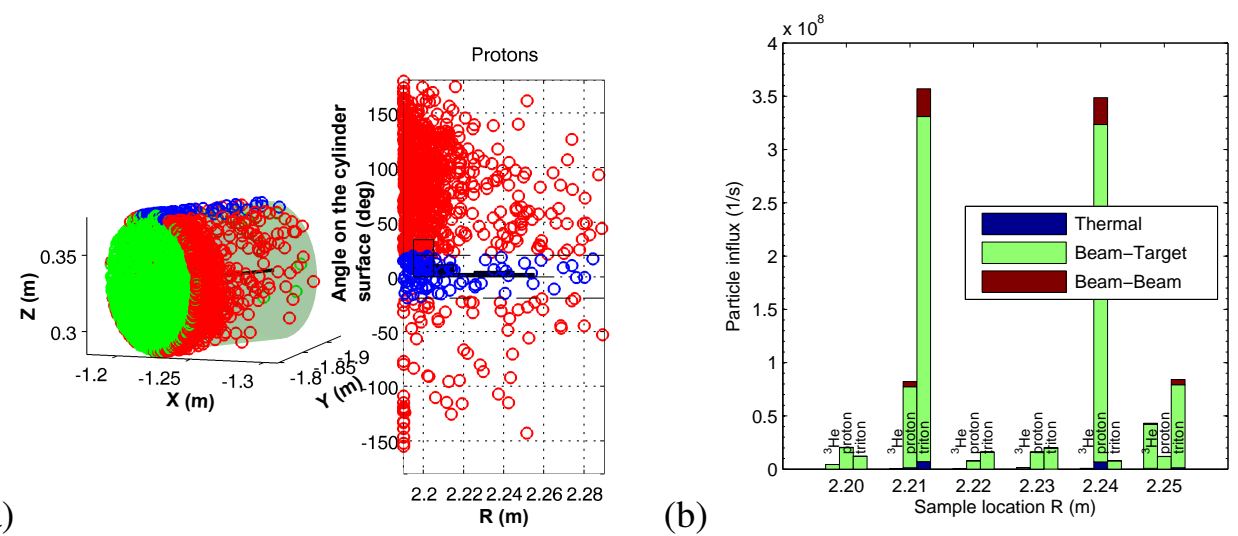

Figure 5: (a) Forward Monte Carlo simulation results [5] indicate that rotating the probe by $90^{\circ}$ would have dramatically increased the flux to the samples. The left panel shows model of the probe graphite cover. Each circle depicts a fusion proton hit. The blue ones are approximately the ones that would enter through the slit and green ones have hit the end of the probe. The right panel shows the side of the graphite cover unwrapped onto a plane. (b) Adjoint Monte Carlo simulated flux to the samples in case of rotating the probe $90^{\circ}$ towards clockwise direction as viewed from top. In each group of bars, the left one is ${ }^{3} \mathrm{He}$, the middle one protons and the right one tritons.

\section{Acknowledgments}

This work was partially funded by the Academy of Finland project No. 259675 and Tekes the Finnish Funding Agency for Innovation under the FinnFusion Consortium.

\section{References}

[1] G. Bonheure, et.al, First escaping fast ion measurements in iter-like geometry using an activation probe, in Proc. 40th EPS Conference on Plasma Physics, Europhysics Conference Abstracts, (Espoo, Finland), p. O6.510, 2013.

[2] G. Bonheure, et.al, Experimental investigation of the confinement of $d(3$ he,p $) \alpha$ and $d(d, p) t$ fusion reaction products in JET, Nuclear Fusion 52 (2012), no. 8083004.

[3] E. Hirvijoki, et.al, ASCOT: Solving the kinetic equation of minority particle species in tokamak plasmas, Computer Physics Communications 185 (2014), no. 4 1310-1321, [arXiv:1308.1904].

[4] R. Budny, et.al, Simulations of alpha parameters in a tftr dt supershot with high fusion power, Nuclear Fusion 35 (1995), no. 121497.

[5] S. Äkäslompolo, et.al, ASCOT simulation of fusion product activation probe experiment in ASDEX Upgrade tokamak, in Proc. 40th EPS Conference on Plasma Physics, Europhysics Conference Abstracts, (Espoo, Finland), p. P5.101, 2013.

[6] S. K. Rathgeber, et.al, Estimation of profiles of the effective ion charge at ASDEX Upgrade with Integrated Data Analysis, Plasma Physics and Controlled Fusion 52 (2010), no. 9095008.

[7] O. Asunta, et.al, Modelling neutral beams in fusion devices: Beamlet-based model for fast particle simulations, Computer Physics Communications 188 (2015) 33-46.

[8] S. Äkäslompolo, Adjoint monte carlo calculation of charged plasma particle flux to wall, April, 2015. arXiv:1504.00214. 